\title{
Skin Color Segmentation by Fuzzy Filter Applied to Face Detection
}

\author{
Abbas Nasrabadi $^{1}$ and Javad Haddadnia ${ }^{2}$
}

\begin{abstract}
- robust face detection in complex background is a challenging task. The complexity in such detection systems stems from the variances in image background, view, illumination, articulation, and facial expression. This article is allocated to introducing a new algorithm for face detection. Fuzzy skin color detection and template matching composed our method instrument. At this paper non-skin parts in the pictures omitted firstly and then result passed to template matching section. Easy implementation and high accuracy detection be feature of our work in compare with other works and indicate the superiority of our work.
\end{abstract}

Index Terms - face detection, fuzzy, template matching, mamdani, skin detection

\section{INTRODUCTION}

A human face detection [1,2] system can be used for Video surveillance and identity detection. Various approaches, based on feature abstraction and statistical analysis, have been proposed. Among them, Rowley and Kanade's neural network approach [2], Viola's asymmetric AdaBoost cascading approach [1], and support vector machine (SVM) approach [10] are a few of the leading ones. In the real world, the complex environment associated with the face pattern detection often makes the detection very complicated. Face is a unique feature of human. Even twins have slight differences in face. Many researches have been done in this area, including that developed by Rowley and al [3] who propose a neural network system able to detect faces of different sizes and rotations at each image position. Osuna [4] uses support vector machine with polynomial kernel to build a face/nonface classifier by maximizing the margin between the two separated classes. Leung and al [5] use a "graph matching" method to find probable faces from Detected facial features. These graphs are generated from the obtained features and the true faces are detected among the candidates by random graph matching. Recently, Viola and al [6] propose a face detection method that computes speedily features using an integral image and combine classifiers in a cascade allowing rejecting background regions quickly. Fleuret and al [7] present a coarse-to-fine face detector which is entirely based on edge configurations. This algorithm visits a hierarchical partition of the face pose space, and in order to

Abbas Nasrabadi is with the Mechatronics Engineering Department, Tarbiat Moallem University of Sabzevar, Sabzevar, Iran, abbas.nasrabadi@gmail.com

Javad Haddadnia is with the Electrical Engineering Department, Tarbiat Moallem University of Sabzevar, Sabzevar, Iran, jhaddadnia@yahoo.com declare one detection, a chain of classifiers from the root to one leaf is found. Their learning approach is purely statistical.

In this paper, human face detection implemented based on fuzzy skin color detection and template matching. The simulation results on the image information bank show that this method has not only good resolution, but also a high resistance against noise. In continuation face situation is introduced using introducing of skin model. In next sections, recommended method of face detection using combinative template matching method and color space changes and based on fuzzy Gaussian color filter.

\section{SKIN COLOR MODEL}

Human skin color has been used and proven to be an effective feature for face detection, localization and tracking. Although different people have different skin color, several studies have shown that the major difference lies largely between their intensity rather than their chrominance [8],[9].Many techniques [10],[3] have been reported for locating skin color regions in the input image. While the input color image is typically in the RGB format, these techniques usually use color components in other color spaces, such as the HSV or YCbCr formats. That is because RGB components are subject to the lighting conditions which may induce face detection to fail. Among many color spaces, we used $\mathrm{YCbCr}$ components since the luminance information is contained in $\mathrm{Y}$ component, and the chrominance information is in $\mathrm{Cr}$ and $\mathrm{Cb}$. Therefore, the luminance information can be easily observed.

$$
P(Y / W s)=\frac{1}{\left.(2 \pi)^{d / 2} \operatorname{det} \sum_{s}\right)^{1 / 2}} \exp \left(-1 / 2(Y-\overline{\mu s})^{T} \sum_{s}^{-1}(Y-\overline{\mu s})\right)
$$

Here, $\mathrm{Y}$ is a color vector and $\mu s$ and $\Sigma s$ are the distribution Parameters (mean vector and the covariance matrix of skin Class ws, respectively), $\mathrm{d}$ is the dimension of the color vector. In the next section we want to considering fuzzy skin detection base on Gaussian Filter.

\section{GAUSIAN FILTER}

To indicate skin region, a set of images including skin areas is used and skin areas are extracted from images. By applying low pass filter on skin samples, the noise reduces and then each skin image changes into $\mathrm{YCbCr}$ space. Then, the two color elements, $\mathrm{Cb}$ and $\mathrm{Cr}$ are obtained.

In this section, a color filter is introduced for separation skin areas. data station of skin images is considered as a 
cluster that all of its elements are skin. This cluster has a mean and a standard deviation, $\sigma$.

$$
O(i, j)=\exp \left[\frac{\left(C_{b}-\overline{C_{b}}\right)^{2}}{2\left(\alpha \sigma C_{b}\right)^{2}}+\frac{\left(C_{r}-\overline{C_{r}}\right)^{2}}{2\left(\alpha \sigma C_{r}\right)^{2}}\right] * 255
$$

In above equation $\alpha$ indicate fuzzy parameter that compose automatically the skin model cluster bandwidths, hence it's deal be effective at filter accuracy.

\section{FUZZY LOGIC}

Fuzzy Logic was initiated in 1965 [7],[8], by Lotfi A. Zadeh, professor for computer science at the University of California in Berkeley. Basically, Fuzzy Logic (FL) is a multi level logic, that allows intermediate values to be defined between conventional evaluations like true/false, yes/no, high/low, etc. Notions like rather tall or very fast can be formulated mathematically and processed by computers, in order to apply a more human-like way of thinking in the programming of computers [9]. Fuzzy systems is an alternative to traditional notions of set membership and logic that has its origins in ancient Greek philosophy. The precision of mathematics owes its success in large part to the efforts of Aristotle and the philosophers who preceded him. In their efforts to devise a concise theory of logic, and later mathematics, the so-called "Laws of Thought" were posited [10]. One of these, the "Law of the Excluded Middle," states that every proposition must either be True or False. Even when Parminedes proposed the first version of this law (around 400 B.C.) there were strong and immediate objections: for example, Heraclitus proposed that things could be simultaneously True and not True.

It was Plato who laid the foundation for what would become fuzzy logic, indicating that there was a third region (beyond True and False) where these opposites "tumbled about." Other, more modern philosophers echoed his sentiments, notably Hegel, Marx, and Engels. But it was Lukasiewicz who first proposed a systematic alternative to the bi-valued logic of Aristotle [9]. Even in the present time some Greeks are still outstanding examples for fussiness and fuzziness, (note: the connection to logic got lost somewhere during the last 2 mileniums [9]). Fuzzy Logic has emerged as a a useful tool for the controlling and steering of systems and complex industrial processes, as well as for household and entertainment electronics, as well as for other expert systems and applications like the classification of SAR data.

\section{INPUT AND OUTPUT MEMBERSHIB FUnCTIONS}

In this work using two parameters to automatically control the Gaussian filter accuracy $(\alpha)$. This parameters extracted directly from luminance dimension pixels mean and standard deviation respectively at $\mathrm{YCbCr}$ color space.

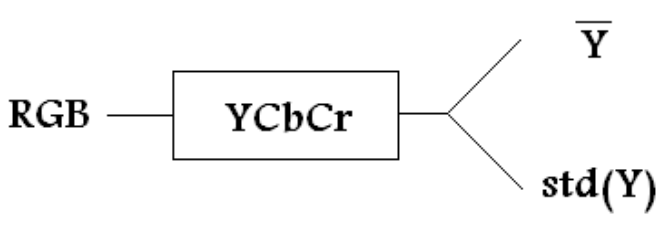

Figure 1. Controlling Parameters extraction

And three membership function determined by practical information about relation between luminance deal and Gaussian filter accuracy as bellow for each parameters,
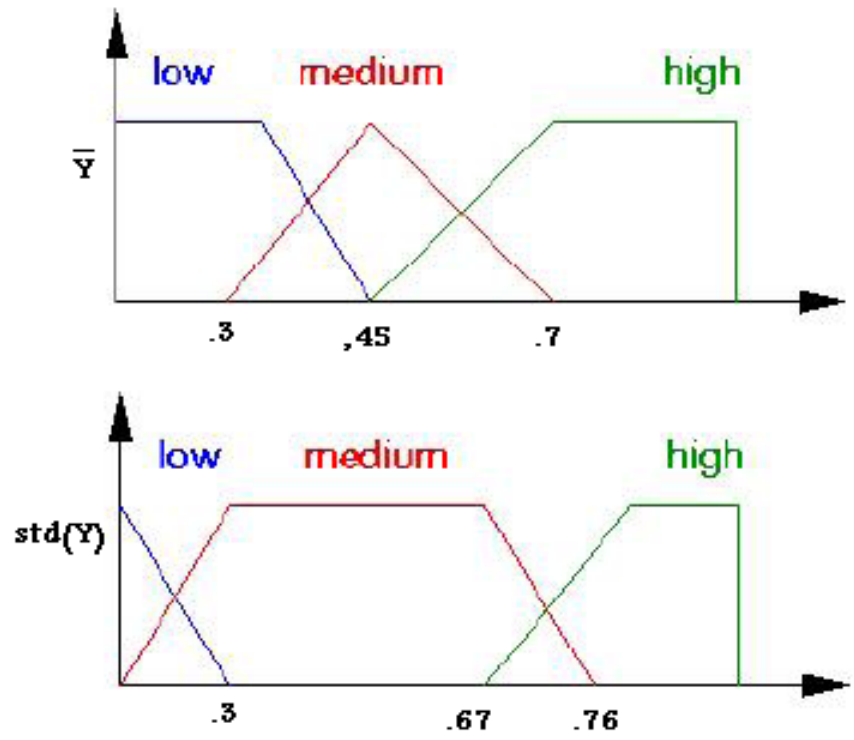

Figure 2. Input membership functions

This functions apply for fuzzification And so proportional output function according practical examination determined as bellow,

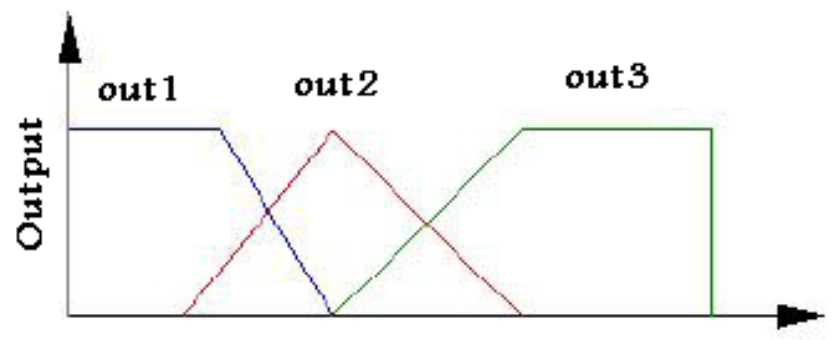

Figure 3. Output membership functions

Above inputs and outputs function relate with together by following some rules. Few rules for sample mentioned in the bellow,

1. if (input1 is low) and (input2 is low) then (output1 is out1) (1)

2. if (input1 is low) and (input2 is medium) then (output1 is out2) (1)

3. if (input1 is medium) and (input2 is medium) then (output1 is out2) (1)

4. if (input1 is high) and (input2 is high) then (output1 is out3) (1)

5. if (input1 is high) and (input2 is medium) then (output1 is out3) (1)

6. if (input1 is low) and (input2 is high) then (output1 is out2) (1)

Following mamdani fuzzy implementation $\alpha$ adjusting according above functions and rules.

Meanwhile defuzzication stage implemented by selecting center of gravity of the fuzzy set as shown for sample in the bellow, 


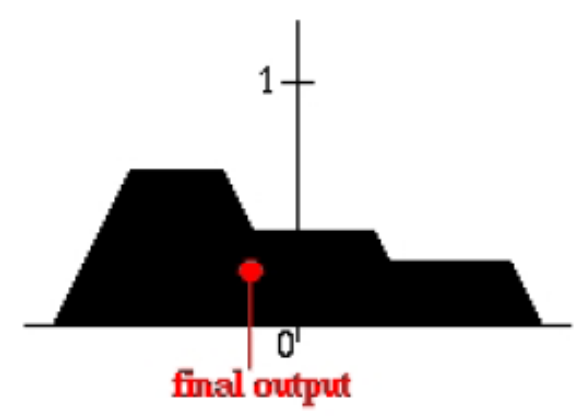

Figure 4. Deffuzification

Final output be $\alpha$ fuzzified deal that adjusted for best accuracy in Gaussian filter.

\section{TEMPlate MATCHING}

The introduced method clusters all the areas of skin and similar to skin in the image. These areas include 3 general areas of skin, related to face, and related to body organs and finally areas close to skin. Figure (5) shows a sample of extracted areas.
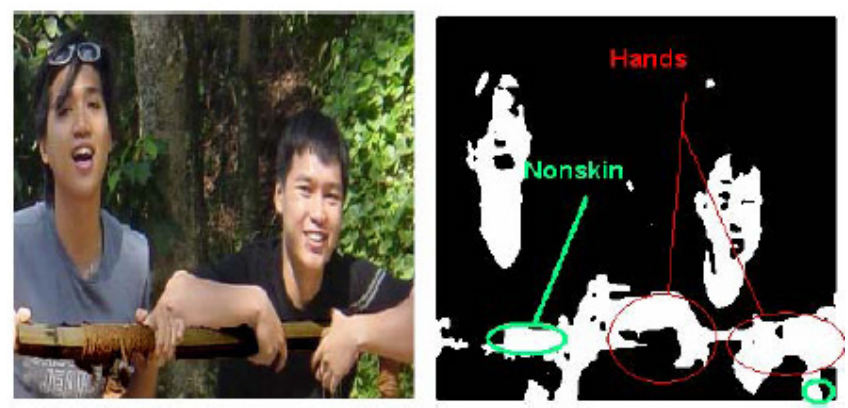

Figure 5. Skin Detection result

In Detection with good quality only the skin area related to face should remain, for this purpose the objective matching test method recognized by a total model from template face. In this method a suitable template is considered for face and this template displaces on the image of created areas. In each displacement the adaptability of this template with image is measured according to correlation. If the adaptability be more than a distinct limit, that area will be considered as skin area of face. The adaptability value can be regulated by a suitable coefficient (R). The used template in this method has been used from the existed model in a special database that is as figure (6).
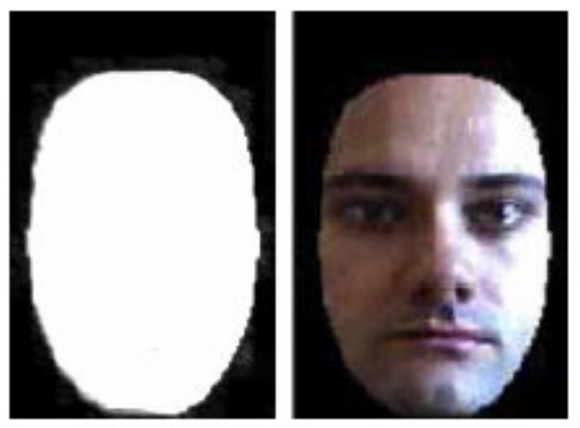

Figure 6. Template

Then we compute the cross-correlation value between the part of the image corresponding to the skin region and the
Template face, a way to determine this value is to use the Following equation:

$$
\begin{gathered}
c o r r=\frac{\sum_{n} \sum_{m}\left(A_{m n}-\bar{A}\right)\left(B_{n m}-\bar{B}\right)}{\sqrt{\sum_{n} \sum_{m}\left(A_{m n}-\bar{A}\right)^{2}\left(B_{n m}-\bar{B}\right)^{2}}} \\
\bar{A}=\frac{1}{M N} \sum_{n} \sum_{m} A_{n m}
\end{gathered}
$$

$$
\bar{B}=\frac{1}{M N} \sum_{n} \sum_{m} B_{n m}
$$

Where $A_{m n}$ and $B_{n m}$ are respectively matrices of a human face template and detected skin region. From our experiments, an optimal threshold value of 0.6 was found to work quite well. Therefore, to classify a region as a face, the resulting correlation value must be greater than 0.6 . The final product of the original color image is displayed with rectangles showing each of the detected faces in the image as represented in Figure (7).

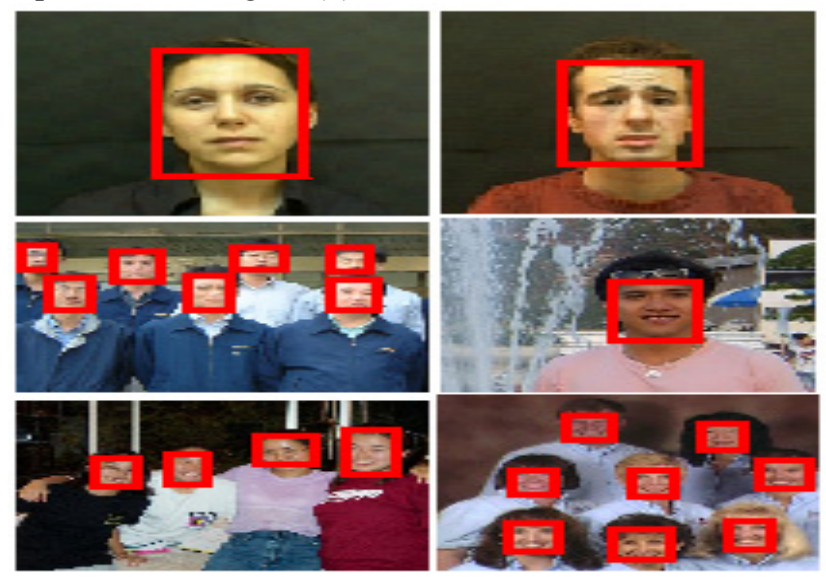

Figure 7. Final Face detection result

\section{CONCLUSION}

We have presented fuzzy approaches to automatic detection of human faces in color images. The proposed approaches Consist of two parts: a human skin segmentation to identify Probable regions corresponding to human faces; and a view based face detection to further identify the location of each human face. The human skin segmentation employs a model based approach to represent and differentiate the background colors and skin colors. The face detection method based on template matching is a promising approach given its satisfactory results. The algorithm retains its high performance even in the presence of structural objects like beard, spectacles, mustaches, glasses etc. However, more features with sophisticated algorithm need to be added in order to use it for more general applications. 


\section{REFERENCES}

[1] J.Haddadnia, M.Amintoosi, "Featured Selection in A Fuzzy Student Sectioning Algorithm", Lecture Note Ocn Computer Science, Accepted for publication.2005.

[2] J.Haddadnia, M.Ahmadi," N-Featured Neural Network Human Face Recognition", Image Vision Computing, 2004.

[3] M.H.Yang, J. Kriegman and N. Ahuja,"Detecting Faces in Images:A Survey,"IEEE Trans. on Pattern Analysis and Machine Intelligence, vol. 24, no. 1, 2002.

[4] E.Hjelmas,B. Kee Low, "Face Detection: A Survey," Computer Vision and Image Understanding 83, 236-274 (2001).

[5] K. Sandeep, A.N. Rajagopalan, "Human face detection in cluttered color images using skin color and edge nformation," Indian Institute of Technology, 2002.

[6] V. Vezhnevets, V. Sazonov, A. Andreeva, "A survey on pixel-based skin color detection techniques," Proc.Graphicon 2003.

[7] L.A. Zadeh, Fuzzy Sets, Information and Control, 1965

[8] L.A. Zadeh, Outline of A New Approach to the Analysis of of Complex Systems and Decision Processes, 1973.

[9] M-H Yang, D. Kriegman and N. Ahuja, ’Detecting Face in Images:A Survey", IEEE Transaction on Pattern Analysis and Machine Intelligence, vol. 24, no. 1, January, 2002, pp. 34-58.

[10] E. Hjelms and B-K Low, 'Face Detection: A Survey", Computer Vision and Image Understanding, vol. 83, 2001, pp. 236-274.

[11] H. Rowley, S. Baluja and T. Kanade, "Neural network-based face detection", In IEEE Trans on PAMI, vol. 20, no. 1, 1998, pp.23-38. 